\title{
Time in Neurogenesis: Conservation of the Developmental Formation of the Cerebellar Circuitry
}

\author{
Eneritz Rueda-Alaña ${ }^{a, b} \quad$ Fernando García-Moreno a, b, c \\ ${ }^{a}$ Achucarro Basque Center for Neuroscience, Scientific Park of the University of the Basque Country (UPV/ \\ EHU), Leioa, Spain; ${ }^{\text {b}}$ Department of Neuroscience, Faculty of Medicine and Odontology, UPV/EHU, Leioa, Spain; \\ 'IKERBASQUE Foundation, Bilbao, Spain
}

\section{Keywords}

Birthdating · Chick - Gecko · Neurodevelopment .

Comparative neuroanatomy $\cdot$ Brain evolution

\begin{abstract}
The cerebellum is a conserved structure of vertebrate brains that develops at the most anterior region of the alar rhombencephalon. All vertebrates display a cerebellum, making it one of the most highly conserved structures of the brain. Although it greatly varies at the morphological level, several lines of research point to strong conservation of its internal neural circuitry. To test the conservation of the cerebellar circuit, we compared the developmental history of the neurons comprising this circuit in three amniote species: mouse, chick, and gecko. We specifically researched the developmental time of generation of the main neuronal types of the cerebellar cortex. This developmental trajectory is known for the mammalian cell types but barely understood for sauropsid species. We show that the neurogenesis of the GABAergic lineage proceeds following the same chronological sequence in the three species compared: Purkinje cells are the first ones generated in the cerebellar cortex, followed by Golgi interneurons of the granule cell layer, and lately by the interneurons of the molecular layer. In the cerebellar glutamatergic lineage, we observed the same conservation of
\end{abstract}

neurogenesis throughout amniotes, and the same vastly prolonged neurogenesis of granule cells, extending much further than for any other brain region. Together these data show that the cerebellar circuitry develops following a tightly conserved chronological sequence of neurogenesis, which is responsible for the preservation of the cerebellum and its function. Our data reinforce the developmental perspective of homology, whereby similarities in neurons and circuits are likely due to similarities in developmental sequence.

(c) 2021 S. Karger AG, Basel

\section{Introduction}

The cerebellum is a conserved feature of vertebrate brains, mostly involved in sensory-motor processing [Buckner, 2013]. It forms at the most anterior region of the hindbrain, surrounded and influenced by the molecular boundaries of the caudal mesencephalon, the isthmus, and the hindbrain [Butts et al., 2014]. It displays one the most elaborated internal structures of the central nervous system, as it contains the most abundant neuronal population of the entire brain, the cerebellar granule cells [Herculano-Houzel et al., 2014]. According to this vast elaboration, and the disparity of environments vertebrates have adapted to, the cerebellum shows a remark- 


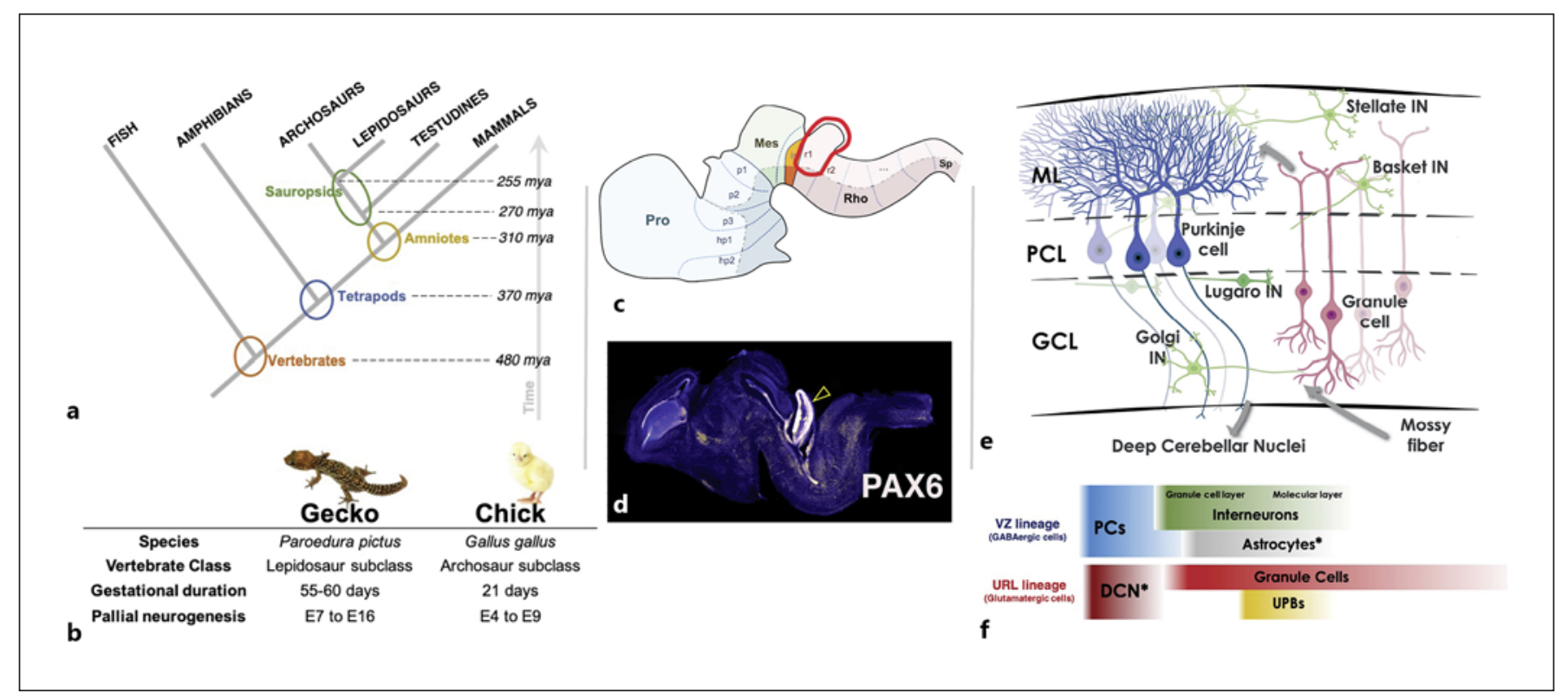

Fig. 1. The developing cerebellum in amniotes. a Phylogenetic tree of amniotes representing the extended period of separate evolution between the different amniote taxa. $\mathbf{b}$ Relevant developmental features of the species under comparison in this study. c Location of the cerebellar primordium in the scheme of a representative amniotic brain. Brain regions are presented following current neuromeric view of the developing brain. $\mathbf{d}$ The cerebellar primordium in a gecko embryo (E32, top), stained with PAX6, in gold. Blue

able degree of morphological variation [Nieuwenhuys et al., 1998]. This variation not only ranges among vertebrate classes, but also within closely related species [Macrì et al., 2019]. Its neuronal extent, functional hierarchical position, and morphologies are among the most diverse across vertebrates [Striedter, 2004]. However, its main cellular types and basic circuitry are astonishingly conserved across species (Fig. 1) [Butler and Hodos, 2005; Hashimoto and Hibi, 2012]. At a glance, it seems that brain evolution found an efficient cerebellar circuitry before the split of amniotes, and this circuit was multiplied differently across species. This way, some cerebella are more structurally intricate than others, but in their functional essence they all process information through a mainly invariable canonical cerebellar circuit [Sillitoe and Joyner, 2007]. We hypothesize that this preservation of the main cell types and connections must be a product of profound developmental conservation.

Two are the main neuronal cell types within the cerebellar cortices of amniotes according to their neurotransmitter phenotype: GABAergic and glutamatergic neurons. The disparity of their neurotransmitter behavior is shows counterstain with DAPI. e Location of the main neuronal types present in the mammalian cerebellar cortex. Arrows represent the direction of the information flow. $\mathbf{f}$ Chronological birthdate of the main neuronal populations in the mammalian cerebellar cortex. Adapted from several neuronal templates by BioRender. com (2020). Retrieved from https://app.biorender.com/biorender-templates. Asterisks mark populations not researched in the article. For nomenclature, please refer to the list of abbreviations.

a product of their divergent developmental origin (Fig. 1e): GABAergic cells, mainly Purkinje cells and GABAergic interneurons, are generated at the ventricular zone (VZ) of the fourth ventricle. Neural stem cells of this VZ express Ptfla and Ascll transcription factors [Hoshino et al., 2005; Sudarov et al., 2011], which direct their cellular lineage towards a GABAergic phenotype. On the contrary, glutamatergic cerebellar cells, such as granule cells and unipolar brush cells, are generated in the upper rhombic lip (URL) [Alder et al., 1996; Wingate, 2001]). This independent germinative zone expresses Atoh1, leading its cellular lineage towards a glutamatergic phenotype [Ben-Arie et al., 1997; Machold and Fishell, 2005]. Beyond the instructions imprinted by the region of the brain on which neurons are generated, the developing time of neurogenesis determines what specific cell types are generated (a feature common throughout the entire CNS [Angevine and Sidman, 1961; Leto et al., 2016; Sagner et al., 2020; Altman and Bayer, 1978]). It is known that in mammals both the germinative cerebellar regions $\mathrm{VZ}$ and URL follow a temporal sequential production of neurons [reviewed in Essen et al., 2020] (Fig. 1e). Briefly, 
Purkinje cells are the earliest neurons generated in the cerebellar cortex [Hashimoto and Mikoshiba, 2003], and granule cells the last ones [Legué et al., 2016]. In other non-mammalian amniotes, the sequential appearance of immunoreactive markers for each of the populations seems to follow an equivalent pattern [Quesada and Genis-Galvez, 1983; Macrì and Di-Poï, 2020; Hanzel et al., 2019]. However, experiments to directly test the time of development at which each population is generated have not been conducted yet.

Therefore, we asked whether the developmental timing of neurogenesis is conserved in the cerebellum across amniote species, as we hypothesize. It has been suggested that heterochronic developmental shifts may underlie cerebellar divergence, even between closely related species. Divergent neurogenic instructions could implement assorted developmental programs, which could lead to cerebellar diversity. We investigated the neurogenic timing of cerebellar populations in two sauropsid species, gecko and chick, representative species of lepidosaur and archosaur vertebrate lineages, and compared them to the known developmental instructions of the mammalian cerebellum.

\section{Materials and Methods}

\section{Animals}

All animal experiments were approved by a local ethical review committee and conducted in accordance with personal and project licenses in compliance with the current normative standards of the European Union (Directive 2010/63/EU) and the Spanish Government (Royal Decrees 1201/2005 and 53/2013, Law 32/107). Fertilized hen eggs (Gallus gallus), obtained from Granja Santa Isabel (Córdoba, Spain), were incubated at $37.5^{\circ} \mathrm{C}$ in a humidified atmosphere until the required stages [Bellairs and Osmond, 2014]. The day when eggs were incubated was considered embryonic day (E) 0 . Gecko eggs were harvested from a local breeder colony of Madagascar ground geckoes (Paroedura pictus) at Achucarro (based on the colony at the Department of Ecology of Charles University, Czech Republic). Adult geckoes were maintained on a 12/12-h light/dark and temperature cycle ( 8 a.m. lights on; $28^{\circ} \mathrm{C}$ diurnal temperature, $23^{\circ} \mathrm{C}$ nocturnal temperature) and provided with ad libitum access to food and water. Eggs were incubated at $28^{\circ} \mathrm{C}$ in a low-humidified atmosphere until the required stages [Noro et al., 2009]. The day when eggs were found in the terrarium was considered E0.

\section{In ovo Birthdating}

Manipulation of chick embryos was performed as previously described [García-Moreno et al., 2018]. For gecko eggs, the procedure was equivalent but with adjustments to the smaller size of the eggs and the high fragility of the eggshell. Briefly, eggs were incubated in a vertical position at either 37.5 or $28^{\circ} \mathrm{C}$. 5 -ethynyl-2-deoxyuridine (EdU; ThermoFisher) was provided via intracardiac/ intravenous injection for chick embryos or via intraventricular injection for gecko embryos, using a fine pulled-glass needle. Drops of sterilized HBSS were added to the egg and chick and gecko embryos were incubated until E15/E19 or E32, respectively.

We adjusted the dose of EdU for each species and developmental stages. We aimed to avoid the aberrant generation of isochronic clusters [Striedter and Keefer, 2000; Rowell, and Ragsdale, 2012]. It is known that BrdU toxicity may cause this aberrant neurogenic pattern, and EdU could produce it as well. In addition, we reduced the dose to the minimum, to avoid potential prolonged incorporation of EdU (Fig. 2). Our birthdating study revealed a short period of EdU incorporation into mitotic cells (Fig. 2a-f), no EdU staining in the VZ days after its injection (Fig. $2 \mathrm{~g}, \mathrm{~h}$ ), and, therefore, an effective pulse-labelling. We injected a volume approximately 0.5 $\mu \mathrm{L}$ of different concentrations of EdU: for chick embryos, we injected $2.5-15 \mu \mathrm{g} / \mu \mathrm{L}$. For gecko embryos, due to its vastly lower number of cells in the brain and its slower metabolism compared to chick, we injected from 2.5 to $25 \mathrm{ng} / \mu \mathrm{L}$. EdU presence was revealed after immunostaining following Click reaction technology (Molecular Probes) and a sulfo-Cyanin 5 (Lumiprobe) conjugate fluorophore. At least 3 animals per stage of injection and species were analyzed, as specified in Table 1 .

\section{Tissue Processing}

Chick embryos were transcardially perfused with fixative solution (4\% paraformaldehyde [PFA] diluted in phosphate-buffered saline [PBS] $0.1 \mathrm{M}, \mathrm{pH} 7.3$ ) after full anesthesia by hypothermia. Embryonic gecko brains were fixed by immersion in PFA. Brains were transferred to PBS $6 \mathrm{~h}$ after fixation. Brains were sectioned in either the sagittal or the coronal plane at 50-70 $\mu \mathrm{m}$ thickness in a vibrating microtome (Leica VT1000S).

\section{Immunohistochemistry}

Single and double immunohistochemical reactions were performed as described previously [Rueda-Alaña et al., 2018] using the following primary antibodies: rabbit antibody to PAX6 (Genetex, GTX113241; 1:1,000), mouse antibody to parvalbumin (PV; Sigma, P3088; 1:750), rabbit antibody to calbindin (Swant, CB D$28 \mathrm{k} ; 1: 1,000)$, rabbit antibody to GABA (Genetex, GTX125988, 1:750) and rabbit antibody to histone $\mathrm{H} 3$ - phospho S10 (Abcam ab47297; 1:1,000). For secondary antibodies (all 1:1,000), we used Alexa 568 goat antibody to rabbit IgG (Molecular Probes, A11011), Alexa 488 goat antibody to rabbit IgG (Molecular Probes, A11034), Alexa 488 goat antibody to mouse IgG (Molecular Probes, A11001), and Alexa 568 antibody to mouse IgG (Molecular Probes, A11004).

\section{Imaging and Analysis}

Images were captured using a Zeiss LSM 710 confocal microscope (Carl Zeiss Microimaging). Similar image parameters (laser power, gain, pinhole, and wavelengths) were maintained for images from each brain and adjusted for new specimens as required. The employed fluorophores were DAPI, Alexa 488, Alexa 568, and Cyanin 5. In selected cases, Z-stacks were taken individually for each channel and then collapsed to get maximum intensity projections. For panoramic views of big brain sections, tile-scan images were composed by a Virtual Scanner (3DHistech Ltd) or the confocal microscope. Images were adjusted and analyzed using ImageJ (Image Analysis in Java, NIH) and Adobe Photoshop CS6 (Adobe Systems Inc.). Images were taken from a minimum of 3 animals successfully birthdated. 

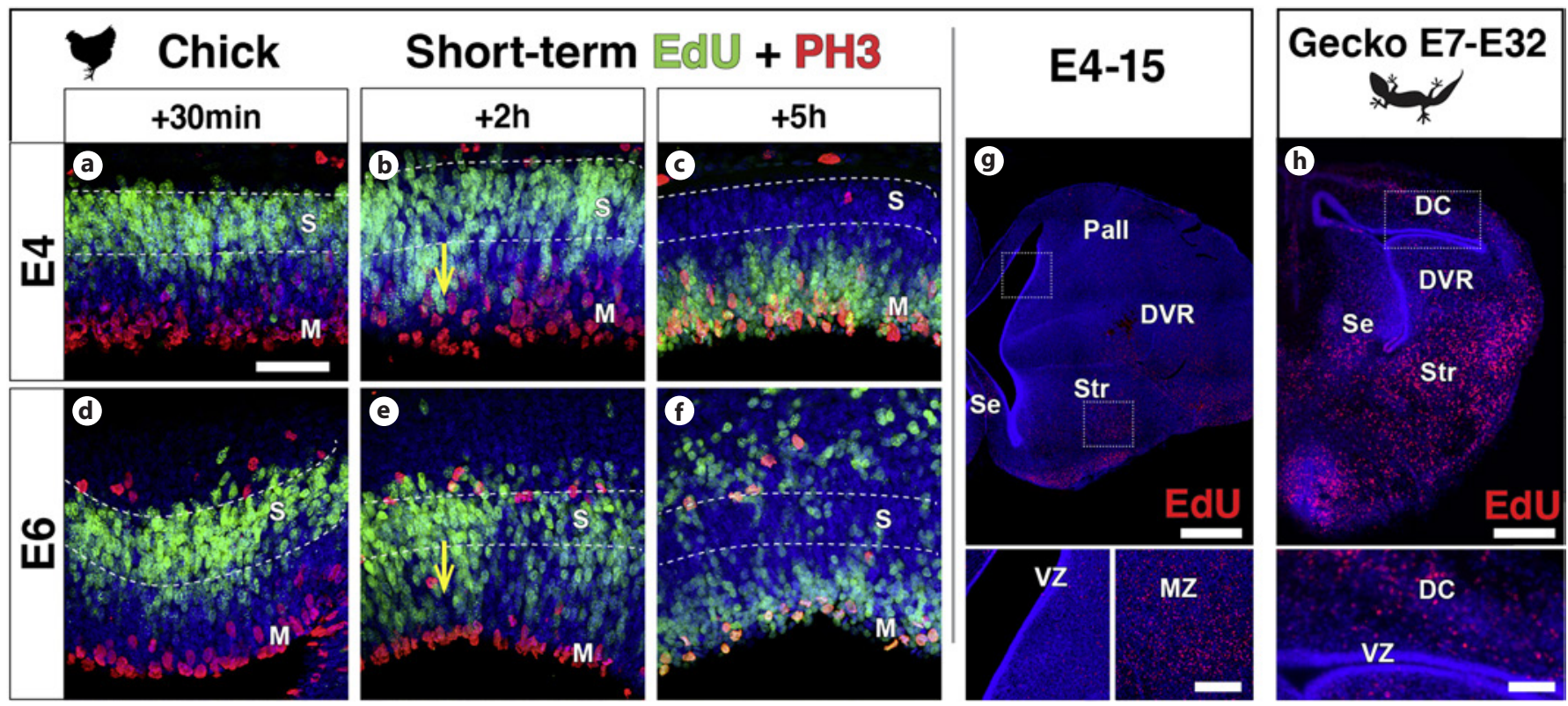

Fig. 2. In ovo EdU administration as an effective birthdating pulselabeling assay. a-f Short-term birthdating of the pallial neurons of the chick at E4 (a-c) and E6 (d-f). Dashed white lines demarcate the region of the neuroepithelium where neural stem cells synthesize new DNA (S-phase, S) in their interkinetic nuclear movement. a, d Thirty minutes after EdU administration, most EdU-labeled cells appear at the basal region of the neuroepithelium, as they are in the S-phase of the cell cycle. $\mathbf{b}, \mathbf{e}$ Two hours after administration of EdU, many EdU cells have already started to descend towards the ventricular surface, following their interkinetic nuclear movement, as marked by the yellow arrow. c, f Five hours after EdU administration, most cells are away from the $S$-phase region of the

neuroepithelium, and have already reached both the ventricular surface and the M-phase of the cycle (M), marked by PH3 immunostaining. The S-phase region appears blank of EdU labeling, as no new EdU is being incorporated at this time after EdU injection. $\mathbf{g}, \mathbf{h}$ A long-term example of EdU incorporation and labeling in chick (g), injected at E4 and researched at E15, and gecko (h), injected at E7 and researched at E31. Coronal sections through the telencephalon, depicting the many labeled neurons within the mantle zones, and the absence of EdU labeling in the VZ cells (insets). For nomenclature, refer to the list of abbreviations. DAPI counterstain in blue. Scale bars, $500 \mu \mathrm{m}(\mathbf{g}), 200 \mu \mathrm{m}(\mathbf{h}), 100 \mu \mathrm{m}$ (g insets), and $50 \mu \mathrm{m}$ (a for a-f and $\mathbf{h}$ inset).

Table 1. Survival of gecko and chick embryos analyzed for this study

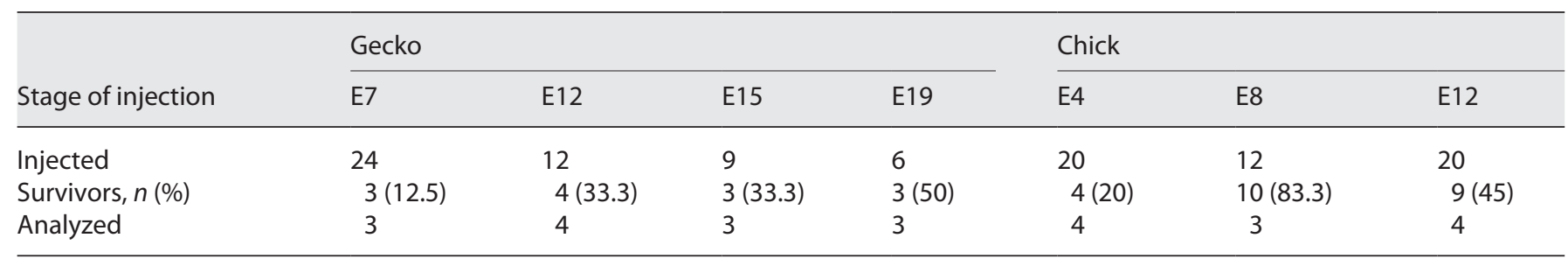

EdU pulse-labeling is a powerful method to describe neurogenic trends and gradients, but not a straightforward method to compare the proportion of cells born at given timepoints between two or more species. There are several critical differences between species, such as the routes of administration, the time that EdU is available for the dividing cells, and the cell cycle duration and features (much longer in gecko than in chick or mouse neural stem cells). Altogether, EdU labels a different fraction of the neurogenic period in each of the species, making the quantitative comparison unreliable.

In some cases, individual pseudo-colored images of equivalent sections from different animals were digitally merged (Fig. 6a).
This way, we obtained a visual representation of the differential distribution of birthdated cells in one single image.

\section{Results}

In search of common and divergent rules of development in the amniote cerebellum, we aimed to research representative species of each main amniote taxon 

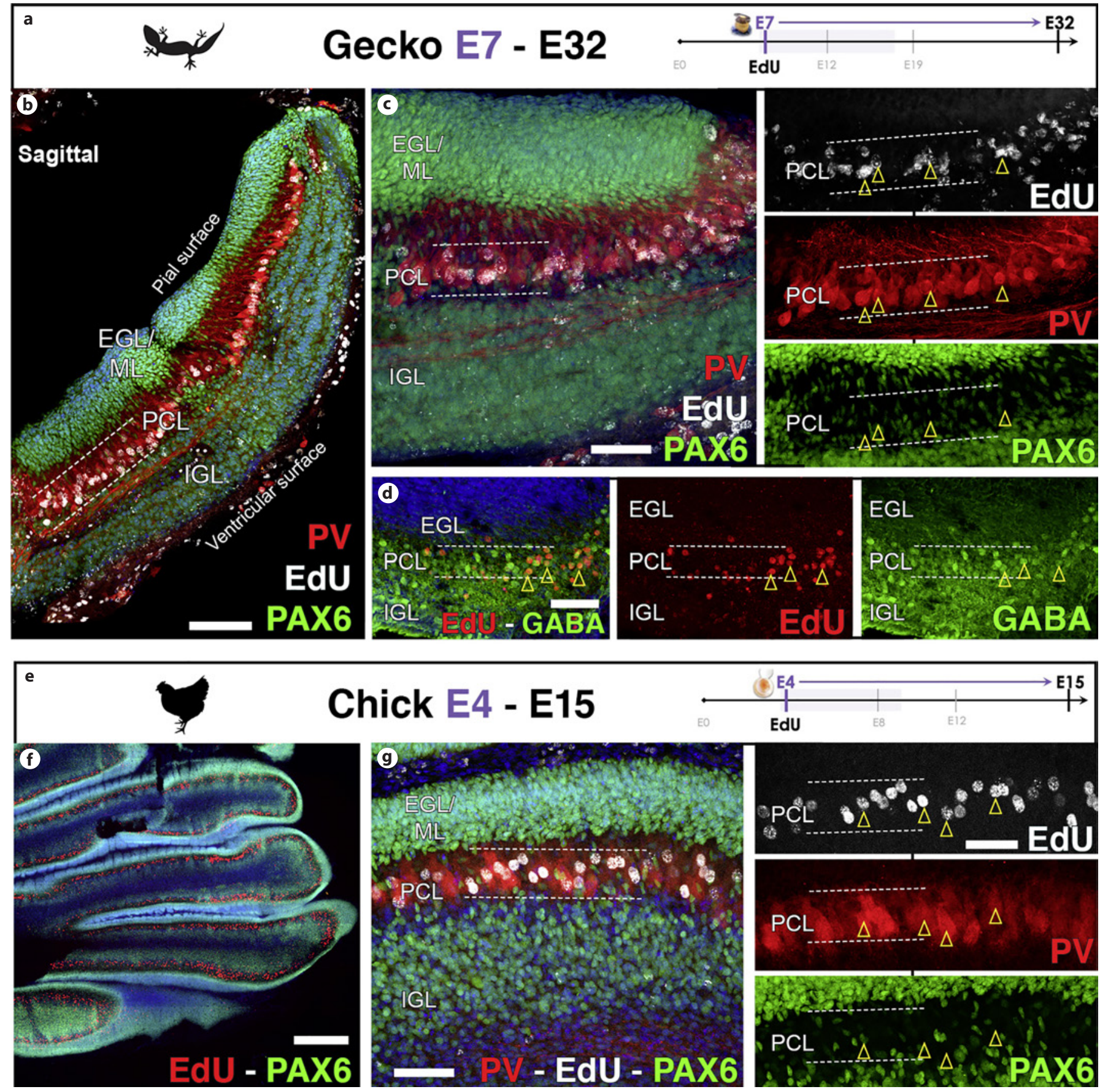

Fig. 3. Early neurogenesis in the sauropsid cerebellum gives rises to Purkinje cells. a-d Birthdating of the cerebellar neurons of the gecko at E7. a Experimental design. Gecko embryos were injected at E7 and harvested at E32. b Sagittal section through the cerebellum showing the lined-up birthdated neurons in the PCL. Immunohistochemistry against PV and PAX6. c High-power view and split channels of the cerebellum in the sagittal section. All cerebellar neurons labelled were Purkinje cells. d Coronal section through a birthdated cerebellum, with immunohistochemistry against GABA. e-g Birthdating of the cerebellar neurons of the chick at E4. e Experimental design. Chick embryos were administered with
EdU at E4 and collected at E15. f Coronal section of the chick cerebellum showing E4 neurons arranged in an organized layer, almost exclusively in the PCL. Immunohistochemistry against PAX6. g High-power view and split channels of the cerebellum in the coronal section. All cerebellar neurons labelled were Purkinje cells, immunoreactive for PV but not for PAX6. For nomenclature, refer to the list of abbreviations. DAPI counterstain in blue. Dashed white lines demarcate the PCL boundaries. Yellow arrowheads indicate representative neurons co-labeled with EdU and the immunohistochemical marker. Scale bars, $500 \mu \mathrm{m}(\mathbf{f}), 100 \mu \mathrm{m}$ (b), and $50 \mu \mathrm{m}(\mathbf{c}, \mathbf{d}, \mathbf{g})$. 
(Fig. 1a). Good-quality descriptive and quantitative data about the development of the mouse cerebellum is available in the literature, including detailed chronological reconstruction of cerebellar development (Fig. 1f) [for a review see Essen et al., 2020]. We compare mouse cerebellar development to two animal representatives of sauropsid species (Fig. 1b). On the lepidosaur taxon, we selected the Madagascar ground gecko (P. pictus, from now on gecko) due to its suitable features for neurodevelopment [Nomura et al., 2013b]. For the archosaur lineage we researched chick cerebellum development (G. gallus, from now on chick), a species on which there is a substantial core of research literature [Quesada and Genis-Galvez, 1983; Alvarez Otero et al., 1993; Karam et al., 2000; Marzban et al., 2010; Hanzel et al., 2019]. The location of the cerebellar anlage during development is conserved throughout the amniote lineage, at the alar portion of the rostral-most region of the rhombencephalon (Fig. 1c). This location is easily identifiable by its early layered cytoarchitectonics and immunohistochemistry pattern (Fig. 1d).

\section{Purkinje Cells Are the Earliest-Generated Neurons in the Sauropsid Cerebellum}

We used the thymidine analogue EdU to birthdate neuronal populations in the developing cerebellum of chicks and geckoes. We used a restricted dose that enabled us to mark isochronically generated cells in a pulselabelling fashion. The EdU was shortly incorporated in the DNA of cycling progenitors (Fig. 2a-f) [García-Moreno et al., 2018], and only labelled the first cohorts of derived neurons, as evidenced by the complete lack of EdU staining in neural stem progenitors days after the administration (Fig. 2g-h).

Fig. 4. Intermediate neurogenesis in the sauropsid cerebellum generates cerebellar GABAergic interneurons and the first glutamatergic neurons. a-f Birthdating of the cerebellar neurons of the gecko at E12. a Experimental design. Gecko embryos were injected at E12 and harvested at E32. b Coronal section through the midbrain and hindbrain of the gecko, showing the location of the cerebellum and the disposition of birthdated cells in the tissue. c Coronal section through the cerebellum showing the location of E12generated neurons. Immunohistochemistry against Purkinje cell markers, PV and CB. On the right, split channels of the rectangle in c, to show the co-labeling of neurons generated at E12 with Purkinje markers at the medial-most region of the cerebellum. $\mathbf{d}$ The cerebellum stained for PAX6. At the bottom, EdU staining showing neurons generated at E12 tend to locate the IGL. e, f Highpower view and split channels of the cerebellum in the coronal section, immunostained for GABA (e) or PAX6 (f). At the medial
The earliest administration of EdU in developing geckoes was performed at E7, a very early stage of brain neurogenesis [Noro et al., 2009; Nomura et al., 2013a]. These injections provided a distinctive staining of linedup, big nuclei at E32. Almost all E7-generated cells in the developing gecko cerebellum were aligned within the Purkinje cell layer (PCL; Fig. 3b-d). Immunohistochemistry against Purkinje cell markers PV (Fig. 3b, c) and neurotransmitter gamma-aminobutyric acid (GABA; Fig. 3b, d) confirmed that early generated neurons in the gecko cerebellum were indeed Purkinje cells.

The equivalent neurogenic stage of chick development is E4 (Fig. 3e). We birthdated E4 cerebellar neurons in chick embryos and found identical results to the gecko experiments (Fig. 3e-g). Nearly all cerebellar neurons generated at $\mathrm{E} 4$ lined up along the chick PCL, throughout all cerebellar folia and the entire cerebellum at E15 (Fig. 3b, c). These early birthdated cells expressed PV, which confirmed their Purkinje cell identity.

Along with known data on mouse Purkinje cell generation [Namba et al., 2011; Sudarov et al., 2011], these results showed that the early birthdate of Purkinje cells is a widely conserved feature across amniote cerebellar cortices.

\section{Neurogenesis of Glutamatergic and GABAergic}

Neurons of the Sauropsid Cerebellum

Next, we assessed the generation of cerebellar neurons at gecko E12 and chick E8 stages (Fig. 4), developmental timepoints after the peak of telencephalic neurogenesis (Tsai et al. [1981] for chick neurogenesis; data not shown for gecko). The latest Purkinje cells of the gecko cerebellum were generated at E12. These late Purkinje cells were located at the medial-most region of the cerebellar cortex at E32 (Fig. 4b, c, e). This fact suggested a spatiotemporal

region of the cerebellum there are some birthdated GABAergic neurons in the PCL in e. $\mathbf{g}-\mathbf{j}$ Birthdating of the cerebellar neurons of the chick at E8. $\mathbf{g}$ Experimental design. Chick embryos were administered with EdU at E8 and collected at E15. $\mathbf{h}$ Coronal section of the chick cerebellum showing E8 neurons located almost exclusively the IGL. Immunohistochemistry against PV. i, j High-power view and split channels of the cerebellar cortex in the coronal section, immunostained for GABA (i) or PAX6 (j). Neurons birthdated at E12 located the IGL and belonged to either the GABAergic (i) or the glutamatergic (j) fate. For nomenclature, refer to the list of abbreviations. DAPI counterstain in blue. Dashed white lines demarcate the PCL boundaries. Yellow arrowheads indicate representative neurons co-labeled with EdU and the immunohistochemical marker. Scale bars, $500 \mu \mathrm{m}$ (b, h), $200 \mu \mathrm{m}$ (c, d), $100 \mu \mathrm{m}$ (f), $50 \mu \mathrm{m}(\mathbf{e}, \mathbf{i}, \mathbf{j})$, and $25 \mu \mathrm{m}$ (insets of $\mathbf{f}, \mathbf{i}, \mathbf{j}$ ).

(For figure see next page.)
38

Brain Behav Evol 2022;97:33-47 DOI: $10.1159 / 000519068$
Rueda-Alaña/García-Moreno 

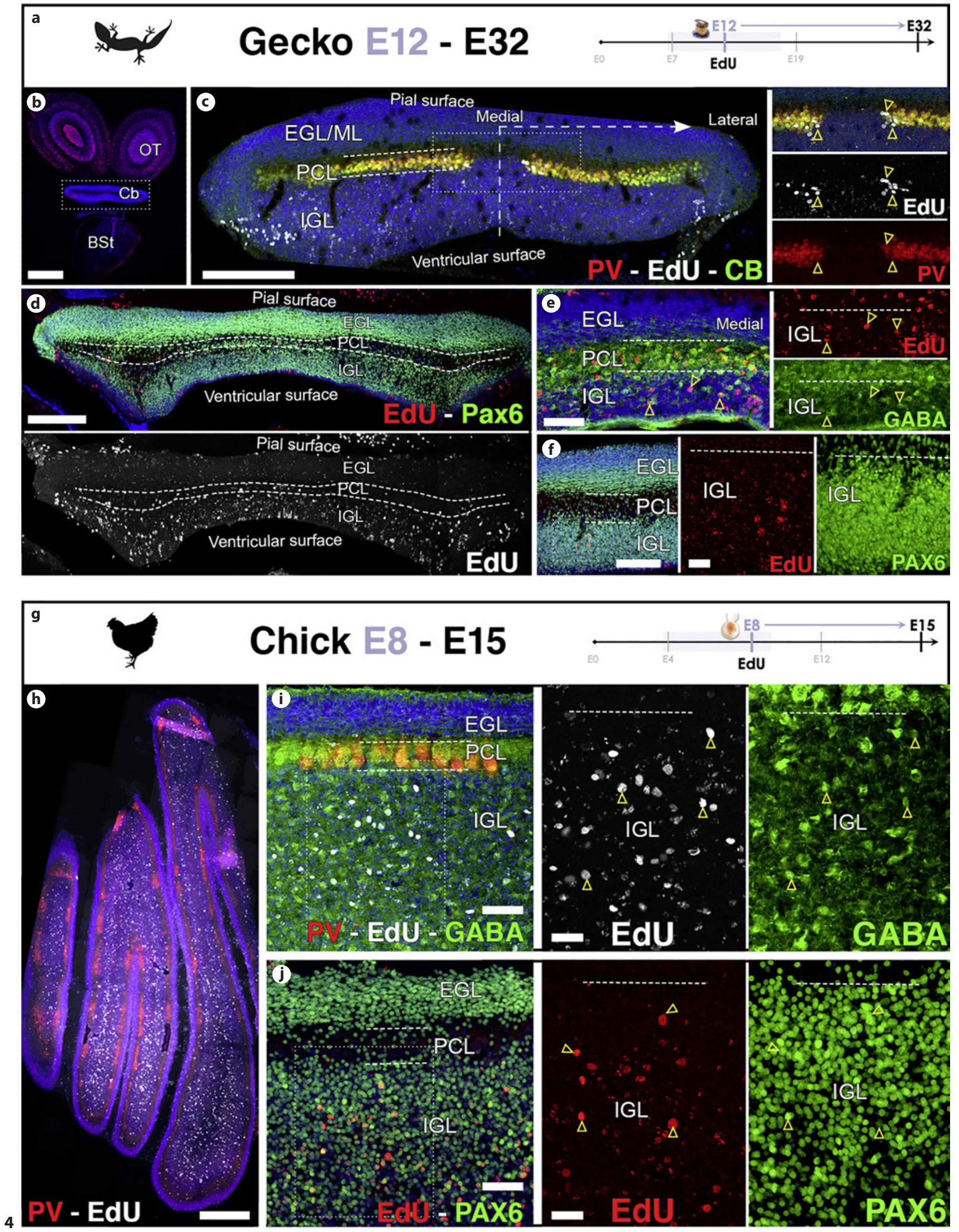
gradient of neurogenesis of the Purkinje cell population, which is also a known feature of mammalian cerebellar development [Namba et al., 2005]. The animals showing this pattern of Purkinje staining also showed scattered EdU staining at the lateral sides of the internal granule cell layer (IGL). In other gecko embryos, we did not observe PCL labelling, but a more extended EdU staining in the IGL (Fig. 4d-f). Sparse, faint EdU labelling was also present in the external granule cell layer (EGL), remnant of the highly mitotic behavior of this germinative compartment (see bottom image in Fig. 4d). E12-generated cells in the IGL belonged to both main neuronal lineages, GABAergic interneurons (Fig. 4e), and PAX6-expressing glutamatergic neurons (Fig. 4f). GABAergic neurons in the IGL may belong to the Golgi subclass, the most common throughout amniotes (Llinas and Hillman [1969] in Butts et al. [2014]). The glutamatergic neurons could be either granule cells or unipolar brush cells, although our immunohistochemical analysis did not allow us to discriminate between both.

The latest Purkinje cells in the chick cerebellum were born at E6 (data not shown). Neurons generated at E8 in the developing chick cerebellum located at E15 the equivalent regions as those generated in the gecko cerebellum at intermediate neurogenic stages (Fig. 4g-j). All neurons birthdated at E8 located the IGL. These neurons, as expected by the gecko birthdating analysis, became both GABAergic interneurons (Fig. 4i) and PAX6+ glutamatergic neurons (Fig. 4j). The glutamatergic cells could belong to either the granule cell or the unipolar brush cell population, although we were not able to discriminate between the two lineages.

Fig. 5. Late neurogenesis in the sauropsid cerebellum generates ML GABAergic cells and granule cells. a-e Birthdating of the cerebellar neurons of the gecko at E15. a Experimental design. Gecko embryos were administered with EdU at E15 and collected at E32. b Coronal section of the gecko cerebellum showing that E15-generated neurons profusely populate the IGL, and some neurons locate the surroundings of the PCL. Immunohistochemistry against PV. c EdU staining of the cerebellar cortex to show the birthdated pattern. d, e High-power view and split channels of the cerebellar cortex in the coronal section, immunostained for PV (i) or GABA (j). $\mathbf{f}-\mathbf{i}$ Birthdating of the cerebellar neurons of the gecko at E19. f Experimental design. Gecko embryos were injected at E19 and harvested at E32. b Sagittal section through the cerebellum of the gecko, showing abundant EdU staining at EGL and IGL. Immunostaining against PV and PAX6. Orange arrows point to granule neurons descending through the PCL towards the IGL. $\mathbf{h}, \mathbf{i}$ Highpower view and split channels of the cerebellum in the sagittal sec-
These data strongly resembled the chronological neurogenesis in the mammalian cerebellum [Zhang and Goldman, 1996; Maricich and Herrup, 1999; Brown et al., 2019]. In the ventricular rhombencephalic lineage, GABAergic interneurons are generated after the production of Purkinje cells. And in the URL lineage, from intermediate stages onwards there is a one-off generation of unipolar brush cells and a constant production of granule cells [Wang et al., 2005] (Fig. 1e, f).

\section{Extended Generation of Granule Cell and Superficial GABAergic Neurons in the Sauropsid Cerebellum}

Mammalian cerebellar neurogenesis persists over developing time, much longer than for any other region in the brain [Miale and Sidman, 1961; Butts et al., 2014]. In the mouse cerebellum, neurogenesis extends up to P21, about 3 weeks after forebrain neurogenesis has ended (with the exceptions of the hippocampus [García-Moreno and Molnár, 2020] and the subventricular zone of the lateral ventricle). We sought for an equivalent extended neurogenic period in the sauropsid cerebellum by EdU administration at late developmental timepoints, when neurogenesis is complete throughout most other brain regions.

First, we assessed gecko neurogenesis at E15 (Fig. 5ae), when most other brain regions have finished their neurogenic process. At E32, we found abundant birthdated neurons in the IGL, as a continuation of the process started at E12 (Fig. 4). Amongst the generated neurons, we identified GABAergic neurons located at the IGL but also cells around Purkinje cells. According to their location, at the bottom of the EGL and future molecular layer $(\mathrm{ML})$, these specific groups of GABAergic interneurons

tion, immunostained for GABA (h) or PAX6 (i). In $\mathbf{h}$, yellow arrowheads point to EdU birthdated cells co-labeled for GABA in the EGL, whereas blue arrowheads point to co-labeled neurons in the IGL. j-n Birthdating of the cerebellar neurons of the chick at E12. j Experimental design. Chick embryos were administered with EdU at E12 and collected at E19. k Coronal section of the chick cerebellum showing that E12 neurons located profusely the EGL and IGL. Immunohistochemistry against CB. I-n High-power view and split channels of the cerebellar cortex in the coronal section, immunostained for PV and PAX6 (I), CB (m), or PV and GABA (n). Neurons birthdated at E12 became glutamatergic granule cells (PAX6+ cells in I) and ML GABAergic cells $(\mathbf{m}, \mathbf{n})$. For nomenclature, refer to the list of abbreviations. DAPI counterstain in blue. Dashed white lines demarcate the PCL boundaries. Yellow arrowheads indicate representative neurons co-labeled with EdU and the immunohistochemical marker. Scale bars, $500 \mu \mathrm{m}(\mathbf{k}), 200$ $\mu \mathrm{m}$ (b), $50 \mu \mathrm{m}$ (d, e, g-i, I-n), and $25 \mu \mathrm{m}$ (c and $\mathbf{h}, \mathbf{I}$ insets).

(For figure see next page.)
Rueda-Alaña/García-Moreno 
may belong to the Stellate cell subclass [Butts et al., 2014]. Neurogenesis at E19 in the gecko brain only continued in the medial cortex of the pallium [García-Moreno and Molnár, 2020] and the cerebellar cortex (Fig. 5f-i). At this late timepoint there was a vast neurogenesis at the EGL, which produced high numbers of granule cells that migrated throughout the PCL to mature into the IGL
(Fig. 5g). Some GABAergic neurons born at E19 were located both at the EGL/ML and IGL (Fig. 5h), whereas the majority of the late neuronal generation produced PAX6+ granule cells (Fig. 5i).

Neurogenesis in the late developing cerebellum of the chick was similar to the neurogenesis described for the gecko. When we administered EdU at E12, we analyzed
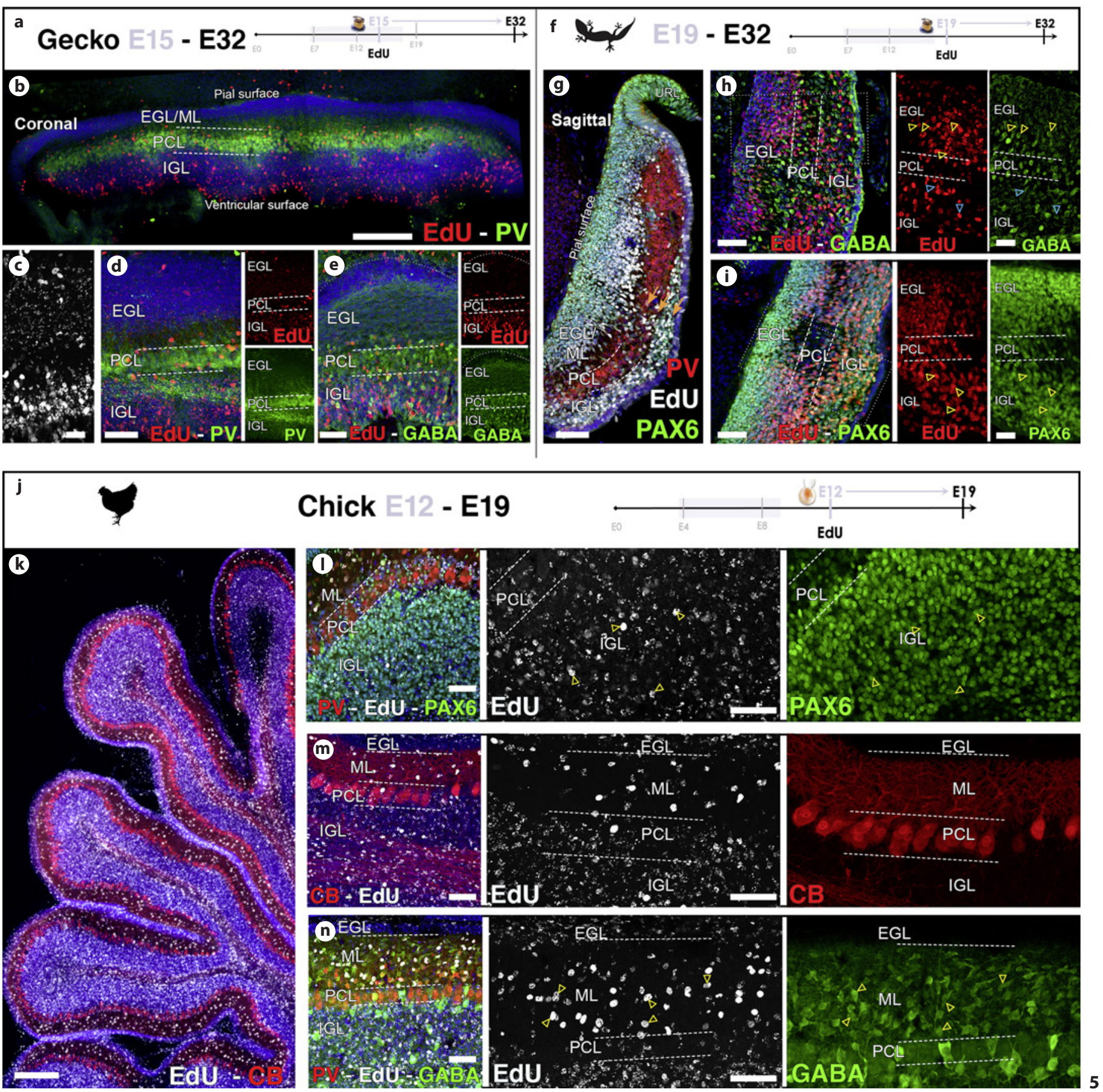

Neurogenesis in Sauropsid Cerebellum

Brain Behav Evol 2022;97:33-47 DOI: $10.1159 / 000519068$ 
the birthdated lineage at E19 (Fig. $5 j-n$ ). In this period of 7 days, birthdated neurons had enough time to be born, migrate, and start their differentiative process. We found massive numbers of neurons generated at this late timepoint (Fig. 5k), the most neurogenically active of all tested stages. Most neurons were granule cells, settling the IGL (Fig. 5l, m). Their EdU staining pattern showed several intensity levels, which correlated with the number of divisions of the EdU+ neural stem cell prior to giving rise to the given labeled neuron. This heterogeneous staining suggested that granule cells were generated at E12 and subsequent stages, until the EdU mark ran out of the mitotically active precursor population. At E19, we also identified strongly stained EdU cells at the ML (Fig. 5m, n). These were GABAergic neurons (Fig. 5n), and according to their bright staining, were born shortly after the EdU injection at E12. Their widespread dispersion through the ML and occasional expression of PV (Fig. 5n) suggested that these neurons were Stellate and Basket neurons (Llinas and Hillman [1969] in Butts et al. [2014]).

The two independent neurogenic processes which occurred at late timepoints of the sauropsid cerebellar development were equivalent to the mammalian case. The late neurogenesis of the ML GABAergic neurons both in chick and gecko cerebella closely resembled the neurogenesis of homologous neurons in the cerebellum of the mouse [Sudarov et al., 2011]. Regarding glutamatergic neurogenesis, we also identified a wide amniote conservation of the extended neurogenesis giving rise to granule cells. This similarity in granule cell neurogenesis suggested that the high numbers of these cells are reached through a conserved prolongation of their neurogenic period in all species.

\section{Discussion}

The cerebellum stands out as one of the most highly conserved structures of the vertebrate brain, at cellular and circuitry level. Here we show that this strong conservation was achieved by a tight preservation of the neurogenic process that generates the cerebellum across amniotes (Fig. 6). From the earliest generated Purkinje cells to the latest populations of glutamatergic granule cells, the sequential order of neuronal production for each of the major cerebellar neuronal classes is the same in mouse, chick, and gecko. Our results, directly assessing comparative cerebellar birthdating, evidence that the developmental program of cerebellar neurogenesis has been conserved for the whole amniote radiation.

\section{Homochrony of Cerebellar Neurogenesis}

A powerful evolutionary tool for generating diversity is developmental heterochrony, i.e., the shift of developmental timing which produces divergent phenotypes [Gould, 1977]. In the case of the brain, heterochronic neurogenic events could be responsible for the diversification of neuronal types and circuits [García-Moreno and Molnár, 2020]. Within the cerebellum, one could argue that the notable degree of morphological variation displayed by amniotes [Nieuwenhuys et al., 1998] could be a product of heterochronic cerebellar development. As an example, the prolonged production of mammalian granule cells [Altman, 1972; Nakashima et al., 2015] could be a mammalian novelty, selected for the generation of highly dense granule cell layers. However, our data and previous research by other authors [Hanaway, 1967; Alvarez Otero et al., 1993; Butts et al., 2011; Hanzel et al., 2019; Macrì and Di-Poï, 2020] show that no significant heterochrony in neurogenesis contributed to the diversification of the cerebellum across amniotes. The vertebrate cerebellum develops following a tight homochrony of neurogenesis in all species tested. This homochrony might be the fundamental feature supporting the unparalleled conservation of the cerebellar circuit.

\section{Conserved Sequential Generation in the GABAergic Lineage}

Among the two main neurotransmitter identities of the cerebellum, the GABAergic lineage is the most diverse. The principal projection neurons of the cerebellar cortex, Purkinje cells, are GABAergic. These neurons are accompanied by a range of GABAergic interneurons that contribute to the modulation of the physiological activity of the circuit [reviewed in Wang and Zoghbi [2001]. The diversity of GABAergic interneurons seems to reach a peak in mammals and birds, and is less rich in reptiles and other vertebrates [Llinas and Hillman, 1969]. However, no matter how diverse this GABAergic lineage is, the developmental trajectory of its populations appears conserved across amniotes.

Purkinje cells are amongst the first neurons generated in the mammalian brain [Altman and Bayer, 1978]. They are surely the first ones appearing in the mammalian cerebellar cortex [Hashimoto and Mikoshiba, 2003; Namba et al., 2011]. To our knowledge, in the case of sauropsids, there was no direct evidence of such early neurogenesis for the Purkinje population. Recently, it was shown that Purkinje cells were the first neurons identifiable by immunohistochemistry in the developing cerebellum of the squamates dragon lizard and house snake [Macrì and Di-
Rueda-Alaña/García-Moreno 
Fig. 6. Comparative chronological construction of the cerebellar cortical circuit in amniotes. a Pseudo-colored photo montages representing the chronological birthdate of the gecko and chick cerebella. Several selected photos, one per experimental stage, were pseudo-colored and merged into the same photo to represent the location of different birthdated cells within the same image. $\mathbf{b}$ Comparative birthdating of cerebellar neuronal populations in three amniote species. Asterisks mark populations not researched in the article. At the bottom, scaled developmental time windows for each species. c Diagram representing the specific birthdates of the neurons in the cerebellar circuit in amniotes. Adapted from several neuronal templates by BioRender.com (2020). Retrieved from https://app.biorender.com/biorendertemplates.
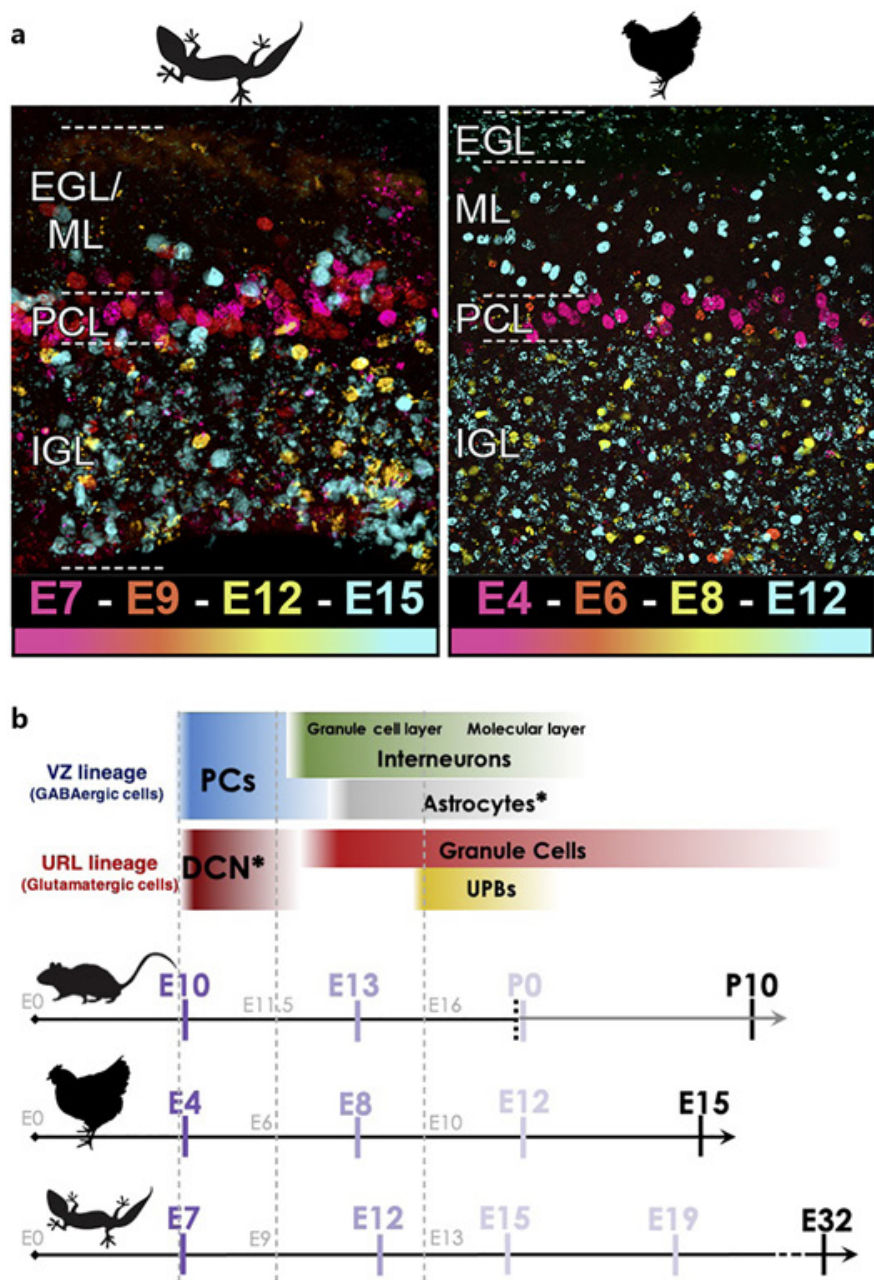

C

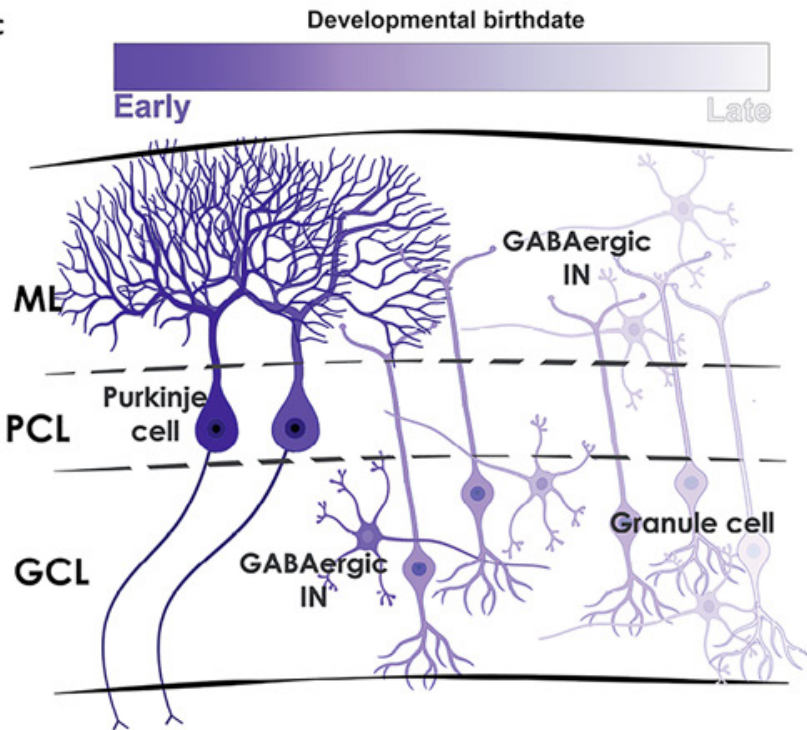


Poï, 2020]. However, their specific birthdate was not assessed. Our birthdating analysis demonstrates that Purkinje cells are the first neurons to be born in the cerebellar cortex of all amniotes.

In mammals, the rest of the GABAergic lineage is generated following an inside-out gradient of neurogenesis. Golgi interneurons of the granule cell layer are generated first, followed by the basket cells of the deep ML, and finally by the stellate cells of the superficial ML [Altman and Bayer, 1978; Sudarov et al., 2011]. Birds do possess homologous basket and stellate interneurons, whereas reptiles seem to lack the stellate type of interneuron [Llinas and Hillman, 1969]. Our birthdating analysis showed the same inside-out temporal order of generation of these populations. Both in chick and gecko cerebella, the first GABAergic interneurons generated reside the granule cell layer, and are lately followed by interneurons at various strata of the ML.

The conservation of the sequential GABAergic generation might predate the split of amniotes, possibly including all the vertebrate subphylum. By researching the differentiation of Purkinje cells in zebrafish, Hamling et al. [2015] concluded that this population is the first one to develop and mature in the cerebellum. In addition, the authors described a spatial regulation of Purkinje cell neurogenesis and differentiation, similar to the known gradient in mammals [Namba et al., 2011] and the case shown here in reptiles. Therefore, critical developmental rules of cerebellar formation, such as timing of neurogenesis and spatial gradients of formation, might be highly conserved across vertebrates.

\section{Conserved Extended Neurogenesis of Glutamatergic Populations}

The most distinctive feature of the glutamatergic neurogenesis in the cerebellum is its protracted duration in developing time. Observations by early neuroscientists already noticed the abundancy of granule neurons and their late development. Both features, abundancy and lateness, are tightly linked. During the embryonic formation of the cerebellum, stem progenitors of the glutamatergic lineage divide and self-renew at the external granule layer [Wang and Zoghbi, 2001; Nakashima et al., 2015]. This transit amplification implies a massive enlargement of progenitor numbers while the rest of the cerebellar neurons are being generated. And so, the external granule layer comprises a substantial pool of progenitors that keeps dividing for a much longer time than any other developmental progenitor pool, contributing a vast number of granule cells [Herculano-Houzel et al., 2014].
The extended neurogenesis of granule precursors produces the largest neuronal population in the brain [Herculano-Houzel et al., 2014]. Granule cells are so numerous in mammals that they have been thought to wrinkle and shape the cerebellar cortex surface [Legué et al., 2016]. Other non-mammalian species are also known to display an external granule layer [Hamling et al., 2015; Hanzel et al., 2019]. Because the avian cerebellum is also highly gyrified, it is possible that the mitotic activity in the external granule layer is a leading cause of cerebellar foliation.

The existence of a mitotic EGL was recently demonstrated in two reptilian species [Macrì and Di-Poï, 2020]. Our data also evidence a high proliferative capacity of the external surface of the sauropsid cerebellum, leading to a vastly prolonged neurogenic period. Indeed, neurogenesis may extend much further in developmental time than the latest stages that we have tested. This is obvious when comparing the developmental timelines of our three model species (Fig. 6b). Together, these lines of research demonstrate that the neurogenic formation of the cerebellum, including its glutamatergic lineages, is highly conserved in all amniotes. The same neurogenic program, including features that were considered exclusive to mammals and birds [Chaplin et al., 2010; Kani et al., 2010], leads to the formation of the amniote cerebellum. This shows that the transit amplification of granule precursors predates the split of amniotes, and has not substantially changed since. Species which do not have a foliated cerebellum also possess an extended glutamatergic neurogenesis (Macrì and Di-Poï [2020] and our data). Altogether, it provides a good example of how stable and unchangeable the neurogenic program of the cerebellum was during the diversified evolution of amniotes.

A second population of glutamatergic cells, the unipolar brush cells, are known to populate the granule cell layer of mammals and birds [Takács et al., 1999]. These interneurons are known to express the transcription factor TBR2 in the mammalian cerebellum [Englund et al., 2006]. However, we could not detect immunoreactivity against this protein, and therefore were not capable of identifying these cells in sauropsid brains. In the mammalian brain, this population is generated in a short period of development from URL-derived progenitors. In the light of our data, we speculate, first, that an equivalent population of glutamatergic interneurons might exist in the granule cell layer of reptiles. Also, we propose that in both birds and reptiles the time of generation of these cells is equivalent and conserved to the one described for mammals [Englund et al., 2006]. The neurogenic pro-
44

Brain Behav Evol 2022;97:33-47 DOI: $10.1159 / 000519068$
Rueda-Alaña/García-Moreno 
gram has been revealed as so stable that it seems likely that unipolar brush cells and their developmental trajectories are conserved through amniotes as well as the rest of the cerebellar populations. If, on the contrary, they are not present in reptiles, or their developmental history is unique to mammals, this population would become a singular exception of the mammalian cerebellum, a novelty of its cerebellar circuitry.

\section{Conserved Developmental Trajectories and the \\ Homology of Neuronal Circuits}

In the mammalian cerebellum, the sequential order of generation of neuronal populations produces an interesting physiological implication. Sudarov et al. [2011] suggested that each neuron is generated earlier than the neuron that projects to it. This way, deep cerebellar nuclear neurons, the latest station of the cerebellar circuit, are generated earlier than Purkinje cells. Purkinje cells are generated before the generation of all GABAergic interneurons that modulate Purkinje activity. Ultimately, the first neurons of the circuit, the granule cell neurons projecting to all GABAergic cells, are the last-generated neurons of the circuit. In sauropsids, the functional circuit is built upon the same neuronal types and connections. In addition, we show that the sequential generation of the neurons of the cerebellar circuit is conserved in sauropsids. Therefore, it is remarkable that the link between neurogenic time and neuronal function within the circuit has been preserved for the extended evolution of amniotes. The developmental rules responsible for this link have remained elusive to evolutionary change.

The cerebellum stands as a major example of neural conservation. The same fundamental circuitry can be found as far as in teleost fish [Bae et al., 2009; Kani et al., 2010; Hamling et al., 2015]. According to the existence of homologous neuronal types, homologous neuronal connections and, importantly, homologous developmental pathways of its neuronal constituents, it is possible to conclude that the cerebellar circuit is homologous amongst vertebrates. Following the pioneer ideas by Källén and his assertion "the longer the developments of two nuclei are similar, the stricter is the homology between the nuclei" [Källén, 1951], we advocate for a developmental point of view of neural homology. Neural circuits will be as homologous across different species as much as the developmental histories of their comprising neurons are conserved.

\section{Acknowledgements}

We warmly thank Prof. Lukáš Kratochvíl (Charles University, Prague) for his inestimable help and support in the constitution of our gecko breeding colony. We thank the Servicio General de Microscopía Analítica y de Alta Resolución en Biomedicina, SGIker at the UPV/EHU.

\section{Statement of Ethics}

Animal experiments conformed to internationally accepted standards. Sauropsid embryos are not protected by the current European (2010/63 / EU) and Spanish (RD 53/2013) regulations, so no specific licenses or approval of the bioethical committee were required.

\section{Conflict of Interest Statement}

The authors have no conflicts of interest to declare.

\section{Funding Sources}

E.R.-A. holds a predoctoral fellowship from the Basque Government. F.G.-M. holds an Ikerbasque Research Fellowship, Spanish Ministry MICNN PGC2018-096173-A-I00 grant and Basque Government PIBA 2020_1_0057 grant.

\section{Author Contributions}

E.R.-A. and F.G.-M. designed and performed the experiments and data analysis, and interpreted the data. F.G.-M. conceived the project, wrote the manuscript, and provided reagents.

\section{References}

Alder J, Cho NK, Hatten ME. Embryonic precursor cells from the rhombic lip are specified to a cerebellar granule neuron identity. Neuron. 1996 Sep;17(3):389-99.

Altman J. Postnatal development of the cerebellar cortex in the rat. I. The external germinal layer and the transitional molecular layer. J Comp Neurol. 1972 Jul;145(3):353-97.
Altman J, Bayer SA. Prenatal development of the cerebellar system in the rat and the cortex of the cerebellum. J Comp Neurol. 1978;179:23-48.

Alvarez Otero R, Sotelo C, Alvarado-Mallart RM. Chick/quail chimeras with partial cerebellar grafts: an analysis of the origin and migration of cerebellar cells. J Comp Neurol. 1993 Jul;333(4):597-615.
Angevine JB Jr, Sidman RL. Autoradiographic study of cell migration during histogenesis of cerebral cortex in the mouse. Nature. 1961 Nov;192(4804):766-8.

Bae YK, Kani S, Shimizu T, Tanabe K, Nojima H, Kimura Y, et al. Anatomy of zebrafish cerebellum and screen for mutations affecting its development. Dev Biol. 2009 Jun;330(2):406-26. 
Bellairs R, Osmond M. The atlas of chick development. 3rd ed. Amsterdam: Elsevier; 2014.

Ben-Arie N, Bellen HJ, Armstrong DL, McCall AE, Gordadze PR, Guo Q, et al. Math1 is essential for genesis of cerebellar granule neurons. Nature. 1997 Nov;390(6656):169-72.

Brown AM, Arancillo M, Lin T, Catt DR, Zhou J, Lackey EP, et al. Molecular layer interneurons shape the spike activity of cerebellar Purkinje cells. Sci Rep. 2019 Feb;9(1):1742.

Buckner RL. The cerebellum and cognitive function: 25 years of insight from anatomy and neuroimaging. Neuron. 2013 Oct;80(3):80715.

Butler AB, Hodos W. Comparative Vertebrate Neuroanatomy: Evolution and Adaptation. 2nd ed. Hoboken (NJ): John Wiley \& Sons, Inc.; $2005 . \quad$ https://doi.org/10.1002/ 0471733849 .

Butts T, Chaplin N, Wingate RJ. Can clues from evolution unlock the molecular development of the cerebellum? Mol Neurobiol. 2011 Feb;43(1):67-76.

Butts T, Green MJ, Wingate RJ. Development of the cerebellum: simple steps to make a little brain'.Development.2014Nov;141(21):403141.

Chaplin N, Tendeng C, Wingate RJ. Absence of an external germinal layer in zebrafish and shark reveals a distinct, anamniote ground plan of cerebellum development. J Neurosci. 2010 Feb;30(8):3048-57.

Englund C, Kowalczyk T, Daza RA, Dagan A, Lau C, Rose MF, et al. Unipolar brush cells of the cerebellum are produced in the rhombic lip and migrate through developing white matter. J Neurosci. 2006 Sep;26(36):9184-95.

García-Moreno F, Anderton E, Jankowska M, Begbie J, Encinas JM, Irimia M, et al. Absence of Tangentially Migrating Glutamatergic Neurons in the Developing Avian Brain. Cell Rep. 2018 Jan;22(1):96-109.

García-Moreno F, Molnár Z. Variations of telencephalic development that paved the way for neocortical evolution. Prog Neurobiol. 2020 Nov; 194:101865.

Gould SJ. Ontogeny and Phylogeny. Cambridge (Mass.): Belknap Press of Harvard University Press; 1977.

Hamling KR, Tobias ZJ, Weissman TA. Mapping the development of cerebellar Purkinje cells in zebrafish. Dev Neurobiol. 2015 Nov;75(11):1174-88.

Hanaway J. Formation and differentiation of the external granular layer of the chick cerebellum. J Comp Neurol. 1967 Sep;131(1):1-14.

Hanzel M, Rook V, Wingate RJ. Mitotic granule cell precursors undergo highly dynamic morphological transitions throughout the external germinal layer of the chick cerebellum. Sci Rep. 2019 Oct;9(1):15218.

Hashimoto M, Hibi M. Development and evolution of cerebellar neural circuits. Dev Growth Differ. 2012 Apr;54(3):373-89.
Hashimoto M, Mikoshiba K. Mediolateral compartmentalization of the cerebellum is determined on the "birth date" of Purkinje cells. J Neurosci. 2003 Dec;23(36):11342-51.

Herculano-Houzel S, Avelino-de-Souza K, Neves K, Porfírio J, Messeder D, Mattos Feijó L, et al. The elephant brain in numbers. Front Neuroanat. 2014 Jun;8:46.

Hoshino M, Nakamura S, Mori K, Kawauchi T, Terao M, Nishimura YV, et al. Ptfla, a bHLH transcriptional gene, defines GABAergic neuronal fates in cerebellum. Neuron. 2005 Jul;47(2):201-13.

Källén B. Embryological studies on the nuclei and their homologization in the vertebrate forebrain. Gleerup; 1951.

Kani S, Bae YK, Shimizu T, Tanabe K, Satou C, Parsons MJ, et al. Proneural gene-linked neurogenesis in zebrafish cerebellum. Dev Biol. 2010 Jul;343(1-2):1-17

Karam SD, Burrows RC, Logan C, Koblar S, Pasquale EB, Bothwell M. Eph receptors and ephrins in the developing chick cerebellum: relationship to sagittal patterning and granule cell migration. J Neurosci. 2000 Sep;20(17):6488-500.

Legué E, Gottshall JL, Jaumouillé E, Roselló-Díez A, Shi W, Barraza LH, et al. Differential timing of granule cell production during cerebellum development underlies generation of the foliation pattern. Neural Dev. 2016 Sep;11(1):17.

Leto K, Arancillo M, Becker EB, Buffo A, Chiang C, Ding B, et al. Consensus Paper: cerebellar Development. Cerebellum. 2016 Dec;15(6): 789-828.

Llinas R, Hillman D. Physiological and morphological organization of the cerebellar circuits in various vertebrates. In: R. Llinas E (editor). Neurobiology of cerebellar evolution and development. Chicago: American Medical Association; 1969. pp 43-73.

Machold R, Fishell G. Math1 is expressed in temporally discrete pools of cerebellar rhombiclip neural progenitors. Neuron. 2005 Oct; 48(1):17-24.

Macrì S, Di-Poï N. Heterochronic developmental shifts underlying squamate cerebellar diversity unveil the key features of amniote cerebellogenesis. Front Cell Dev Biol. 2020 Oct; 8:593377.

Macrì S, Savriama Y, Khan I, Di-Poï N. Comparative analysis of squamate brains unveils multi-level variation in cerebellar architecture associated with locomotor specialization. Nat Commun. 2019 Dec;10(1):5560.

Maricich SM, Herrup K. Pax-2 expression defines a subset of GABAergic interneurons and their precursors in the developing murine cerebellum. J Neurobiol. 1999 Nov;41(2):281-94.

Marzban H, Chung SH, Pezhouh MK, Feirabend $\mathrm{H}$, Watanabe M, Voogd J, et al. Antigenic compartmentation of the cerebellar cortex in the chicken (Gallus domesticus). J Comp Neurol. 2010 Jun;518(12):2221-39.
Miale IL, Sidman RL. An autoradiographic analysis of histogenesis in the mouse cerebellum. Exp Neurol. 1961 Oct;4(4):277-96.

Nakashima K, Umeshima H, Kengaku M. Cerebellar granule cells are predominantly generated by terminal symmetric divisions of granule cell precursors. Dev Dyn. 2015 Jun;244(6):748-58.

Namba K, Sugihara I, Hashimoto M. Close correlation between the birth date of Purkinje cells and the longitudinal compartmentalization of the mouse adult cerebellum. J Comp Neurol. 2011 Sep;519(13):2594-614

Namba T, Mochizuki H, Onodera M, Mizuno Y, Namiki H, Seki T. The fate of neural progenitor cells expressing astrocytic and radial glial markers in the postnatal rat dentate gyrus. Eur J Neurosci. 2005 Oct;22(8):1928-41.

Nieuwenhuys R, ten Donkelaar H, Nicholson C. The central nervous system of vertebrates. Berlin: Springer; 1998. https://doi. org/10.1007/978-3-642-18262-4.

Nomura T, Gotoh H, Ono K. Changes in the regulation of cortical neurogenesis contribute to encephalization during amniote brain evolution. Nat Commun. 2013a;4(1):2206.

Nomura T, Kawaguchi M, Ono K, Murakami Y. Reptiles: a new model for brain evo-devo research. J Exp Zoolog B Mol Dev Evol. 2013b Mar;320(2):57-73.

Noro M, Uejima A, Abe G, Manabe M, Tamura K. Normal developmental stages of the Madagascar ground gecko Paroedura pictus with special reference to limb morphogenesis. Dev Dyn. 2009 Jan;238(1):100-9.

Quesada A, Genis-Galvez JM. Early development of the granule cell in the cerebellum of the chick embryo. J Morphol. 1983 Dec;178(3):323-34.

Rowell JJ, Ragsdale CW. BrdU birth dating can produce errors in cell fate specification in chick brain development. J Histochem Cytochem. 2012 Nov;60(11):801-10.

Rueda-Alaña E, Martínez-Garay I, Encinas JM, Molnár Z, García-Moreno F. Dbx1-derived pyramidal neurons are generated locally in the developing murine neocortex. Front Neurosci. 2018 Oct; $12: 792$.

Sagner A, Zhang I, Watson T, Lazaro J. Temporal patterning of the central nervous system by a shared transcription factor code. bioRxiv. 2020. doi: https://doi.org/10.1101/2020.11. 10.376491 .

Sillitoe RV, Joyner AL. Morphology, molecular codes, and circuitry produce the complexity of the cerebellum. Annu Rev Cell Dev Biol. 2007;23:549-77.

Striedter GF. Principles of brain evolution. Sinauer; 2004.

Striedter GF, Keefer BP. Cell migration and aggregation in the developing telencephalon: pulse-labeling chick embryos with bromodeoxyuridine.JNeurosci.2000 Nov;20(21):802130. 
Sudarov A, Turnbull RK, Kim EJ, Lebel-Potter M, Guillemot F, Joyner AL. Ascll genetics reveals insights into cerebellum local circuit assembly. J Neurosci. 2011 Jul;31(30):11055-69.

Takács J, Markova L, Borostyánköi Z, Görcs TJ, Hámori J. Metabotrop glutamate receptor type 1a expressing unipolar brush cells in the cerebellar cortex of different species: a comparative quantitative study. J Neurosci Res. 1999 Mar;55(6):733-48.
Tsai HM, Garber BB, Larramendi LMH. 3H-thymidine autoradiographic analysis of telencephalic histogenesis in the chick embryo: I. Neuronal birthdates of telencephalic compartments in situ. J Comp Neurol. 1981;198(2):275-92.

van Essen MJ, Nayler S, Becker EB, Jacob J. Deconstructing cerebellar development cell by cell. PLoS Genet. 2020 Apr;16(4):e1008630.

Wang VY, Rose MF, Zoghbi HY. Math1 expression redefines the rhombic lip derivatives and reveals novel lineages within the brainstem and cerebellum. Neuron. 2005 Oct;48(1):3143.
Wang VY, Zoghbi HY. Genetic regulation of cerebellar development. Nat Rev Neurosci. 2001 Jul;2(7):484-91.

Wingate RJ. The rhombic lip and early cerebellar development. Curr Opin Neurobiol. 2001 Feb;11(1):82-8.

Zhang L, Goldman JE. Generation of cerebellar interneurons from dividing progenitors in white matter. Neuron. 1996 Jan;16(1):47-54. 